\title{
ACOUSTIC THERMOAGITATION BASED ON PIEZOELECTRIC $\beta$-PVDF POLYMER FILMS Potential Evaluation in Lab-on-a-Chip Applications
}

\author{
V. F. Cardoso, G. Minas \\ University of Minho, Department of Industrial Electronics, Campus de Azurém, 4800-058, Guimarães, Portugal \\ vanessa@dei.uminho.pt,gminas@dei.uminho.pt \\ P. Martins, J. Serrado Nunes, L. Rebouta, S. Lanceros-Méndez \\ University of Minho, Department of Physics, Campus de Gualtar, 4710-057, Braga, Portugal \\ pmartins@fisica.uminho.pt,jivago@fisica.uminho.pt,rebouta@fisica.uminho.pt,lanceros@fisica.uminho.pt \\ G. Botelho \\ University of Minho, Department of Chemistry, Campus de Gualtar, 4710-057, Braga, Portugal \\ gbotelho@quimica.uminho.pt
}

Keywords: Lab-on-a-chip, PVDF, Acoustic thermoagitation.

Abstract: $\quad$ This paper describes a lab-on-a-chip device with acoustic thermoagitation based on a piezoelectric $\beta$-PVDF polymer. The device is used for testing and monitoring biochemical parameters in biological fluids using optical absorption spectrophotometry. Experimental results regarding the influence of the electrical signal amplitude and frequency applied for the generation of acoustic thermoagitation is presented. The individual contribution of the heating and the microagitation provided by the actuation of the piezoelectric film for the fluids mixture was determined. The paper is completed with a study of the $\beta$-PVDF degradation with transparent conductive electrodes, ITO and AZO, when placed in contact with uric acid fluids. The final goal of using this technique is the improvement of mixing and reaction time without interfering with biochemical reactions and analytical measurements.

\section{INTRODUCTION}

For the development of a lab-on-a-chip device for fluidic analysis, the microfluidics technologies have been a vital tool allowing the fabrication of precise and small structures. The huge interest in these technologies is inherent to the performance achieved: reduction on the sample quantity, high integration and consequently high potential for fluids automation and control in sub-microliter volumes, decrease of response time, reduction of chemical quantities stored and a large reduction in total costs (Auroux et. al, 2002).

Lab-on-a-chip devices can perform several analyses simultaneously. In order to get in-loco, quick and reliable results, they need an automatic system for microfluids control, which can cover all the steps of a chemical or biological process.
Usually, a bio(chemical) process needs the mixture of fluids. For that, devices based in MEMS (Micro Electro Mechanical Systems) such as micropumps (Reyes et. al, 2002) and microvalves (Rife et. al, 2000) are used, but they increase the device cost, need complex control systems and their integration is complex. The mixture of the fluids using just diffusion avoids these disadvantages. However, when large molecules with small diffusivities take part in the reaction, it is needed high transit times of the molecules on the channels and consequently long channels (Ottino et. al, 2004). To overcome this high transit times, it is necessary to develop alternative methods for improving mixture.

The use of acoustic waves is an interesting solution and it is one of the main issues of this present work. The acoustics waves traveling inside the fluid create differential pressures and induce the so called acoustic propagation. For occurring the 
acoustic microagitation of the fluids, the reaction chamber must be coated with an electroactive polymer. One example is the $\beta$-PVDF (LancerosMendez et. al, 2006), which the use for fluid microagitation purposes is the main innovation of this work. Applying an a.c. voltage to the contacts of the piezoelectric $\beta$-PVDF film, it is produce mechanical oscillations promoting the movement, mixture and reaction of the fluids, as well the heat generated by this technique, the so called acoustic thermoagitation.

\section{DEVICE DESCRIPTION}

This paper describes the incorporation and validation of an acoustic thermoagitator based on a piezoelectric $\beta$-PVDF polymer in a fully-integrated disposable lab-on-a-chip for point of care testing and monitoring of biochemical parameters in biological fluids. This lab-on-a-chip has interesting characteristics such as portability, low-cost and disposability. Furthermore, it has a completely automatic operation and uses optical absorption spectrophotometry as analytical measurement technique.

\subsection{Biosystem Operation}

The lab-on-a-chip is composed by two dies: the fluidic die and the detection die (Figure 1).

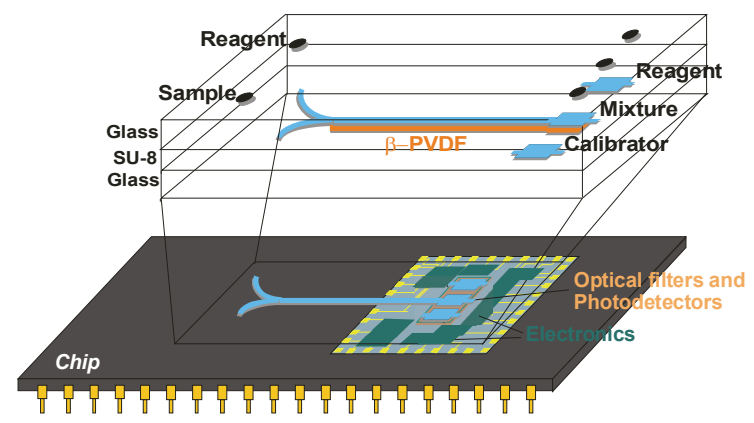

Figure 1: Schematic representation of the lab-on-a-chip structure with the $\beta$-PVDF deposited underneath the microfluidic structure.

The microfluidic die, fabricated in SU-8, includes the microchannels and the reaction chambers. Three reaction chambers are needed for each analysis. One with a well-known concentration sample of the biochemical parameter that is being analysed, is used for the calibration of the device. Other, for the mixture of the sample and the reagent is needed, to perform the analysis of the coloured mixed solution. Finally, a third one is used for the chemical reagent in order to obtain the baseline reference. Underneath the reaction chambers was deposited the piezoelectric $\beta$-PVDF polymer with its corresponding electrodes.

The detection die includes the photodetectors and the electronic components for signal actuation and detection, all fabricated in CMOS technology. Above the photodetectors, there are several highselective band-pass optical filters, deposited by Ion Beam Deposition, that select the wavelength according to the several biomolecules into analysis. This optical filtering system allows the use of noncalibrated external polychromatic light source.

\subsection{Analytical Measurement Technique}

Among the several analytical techniques available in laboratories of clinical analysis, the spectrophotometry is the most used. However, this technique cannot be used directly since a high number of biomolecules for clinical analysis do not have chromophores which absorb the light in the visible spectra. In order to overcome this limitation, several specific chemical reactions allow to transform these biomolecules in colored products which absorbency is within the visible light spectra colorimetric reactions.

In the ideal colorimetric analysis, the mixture coloration intensity is proportional to the concentration of the biochemical parameter and can be quantified measuring the optical absorption of the mixture (reagent + biomolecule) at a specific wavelength (Thomas, 1999).

\subsection{Acoustic Streaming}

The acoustic streaming is a steady flow generated by the propagation of acoustic waves in a viscous fluid. It arises from the transfer of momentum and energy of the acoustic field to the medium, through its acoustic attenuation.

Acoustic streaming offers several distinct advantages for application in microfluidic devices (Frampton et. al, 2004). In this work, the acoustic streaming is due to the absorption of the acoustic energy in the fluid itself. This absorption results in a radiation pressure in the direction of the acoustic propagation and is termed by quartz wind.

Quartz wind velocity and effective pressure are limited by the heating and cavitation tolerance. A small fraction of the incident acoustic energy goes into kinetic energy of the fluid and the rest is transformed into heat. In this way, the acoustic thermoagitation generated by this effect becomes 
advantageous, once in some applications or reactions, the increase of temperature is beneficial in order to reduce the mixture time (Rife et. al, 2000).

\subsection{Poly(vinylidene fluoride)}

Nowadays, with the rapid development of polymer processing methods and synthetic technology, the industrial materials used in various manufacturing fields are being substituted by polymer substances, which have better properties than those of materials previously used in many industrial areas. One of the polymer that has received increased attention is the poly(vinylidene fluoride), also known as PVDF. It shows an unusual polymorphism in this class of materials, showing four different crystalline phases. From the technology point of view, the $\beta$-phase is the one which shows better properties to be applied in sensors, actuators and transducers, due to its higher piezo- piro- and ferroelectric properties.

Moreover, it shows an excellent combination of processability, chemical agent resistance, lightness, moldability and low-cost production. While ceramic materials break easily and have hard and dense structures, PVDF is flexible, has a low density and can be easily produced into thin-films (Brown, 1992). This polymer also shows low acoustic and mechanical impedance (Foster, 2000) crucial for generating the acoustic waves that produce the thermoagitation of the fluids, as is the purpose of this study. Another interesting feature of the PVDF is its transparency. Indeed, in the case of this application, the analytical measurement by spectrophotometry requires that the PVDF and the corresponding conductive electrodes, deposited in the reaction chamber, are transparent to visible light.

\section{EXPERIMENTAL RESULTS}

\subsection{Acoustic Thermoagitation}

The quantitative evaluation of the mixing process was carried out using the Far Diagnostic kit and standards of urine with $5 \mathrm{mg} / \mathrm{dl}$ of uric acid concentration. The reagent reacts with the sample of urine containing uric acid in a 40:1 ratio with a maximum absorption at $550 \mathrm{~nm}$.

The acoustic thermoagitation was studied for various frequencies and amplitudes of the electrical signal applied to the electrical contacts of a $110 \mu \mathrm{m}$ thick $\beta$-PVDF film. The system was calibrated and the reaction was studied up to a maximum time of
20 minutes, which is the time of the complete mixture without thermoagitation.

The thermoagitation was performed powering the $\beta$-PVDF, with a sinusoidal signal of $10 \mathrm{~V}$ amplitude at different frequencies. After, a $10 \mathrm{MHz}$ frequency sinusoidal signal with various amplitudes was applied. They results were processed to obtain the results shown in Figure 2.

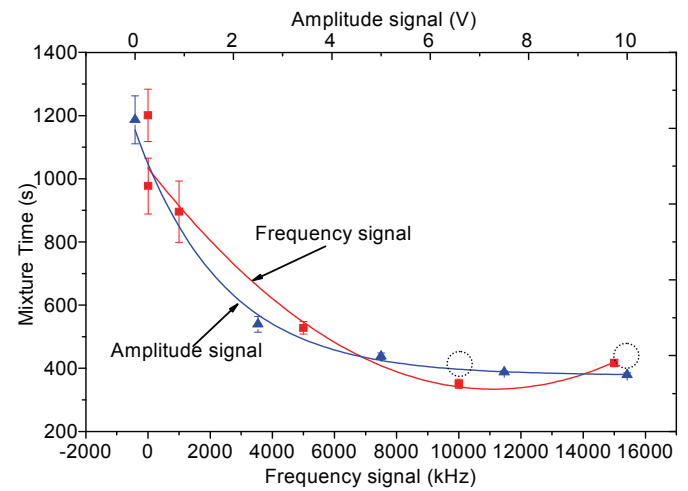

Figure 2: Mixing time as a function of the frequency and amplitude of the signal applied to the electrical contacts of a $110 \mu \mathrm{m}$ thick $\beta$-PVDF film.

It can be observed that with the application of thermoagitation, the mixing occur in a faster way, being the time necessary to obtain the complete mixing, at $10 \mathrm{~V}$ amplitude and $10 \mathrm{MHz}$, only two sevenths $(351 \mathrm{~s})$ of the complete mixing time without thermoagitation $(1200 \mathrm{~s})$. In this way, the resonance frequency of the used $\beta$-PVDF film $(10 \mathrm{MHz})$ is the most efficient for the generation of thermoagitation. This result is consistent with the theory (Eiras, 2007). On the other hand, the mixing time decreases as the signal amplitude increases.

According to previous results, it was determined the individual contribution of the agitation and the heating generated by the use of this acoustic thermoagitation technique. For that, the temperature profile of the sample was measured applying an electrical signal of $10 \mathrm{~V}$ amplitude and $10 \mathrm{MHz}$ to the $\beta$-PVDF film. Then, the sample was heated with the same temperature profile obtained before with the thermoagitation, using a temperature controller (SHIMADZU TCC-260). It was observed that, for the two sevenths in terms of gains achieved by the application of the acoustic thermoagitation technique, three-fifths are due to heating and two fifths to agitation.

\subsection{Transducer Degradation}

As the first application of the present lab-on-a- chip will be for measurement of uric acid concentrations, 
a degradation study (using a high-performance liquid chromatograph model 875 from Jasco) of the $110 \mu \mathrm{m} \beta$-PVDF film with ITO (Indium Tin Oxide) and AZO (Aluminium doped Zinc Oxide) electrodes, when placed in contact with the biological fluids, was performed. The study was done with the Far Diagnostic kit of uric acid, described previously. The chemical reaction was performed with and without acoustic thermoagitation during 20 minutes. The thermoagitation was set using an electrical signal at $10 \mathrm{~V}$ amplitude and $10 \mathrm{MHz}$. The results shown in Figures 3 and 4 are representative for not degraded and degraded electrodes, respectively.

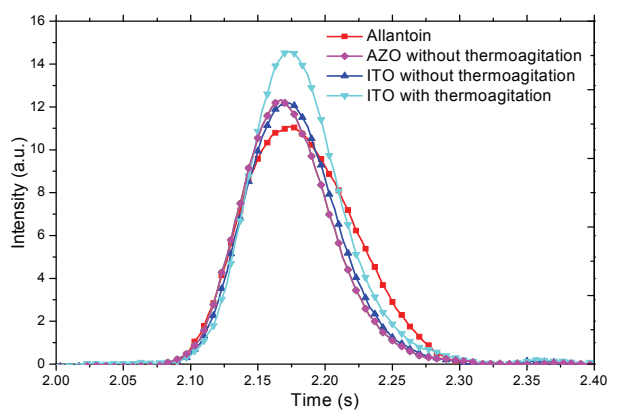

Figure 3: Chromatogram of the samples that did not suffered degradation with a reaction time of $20 \mathrm{~min}$.

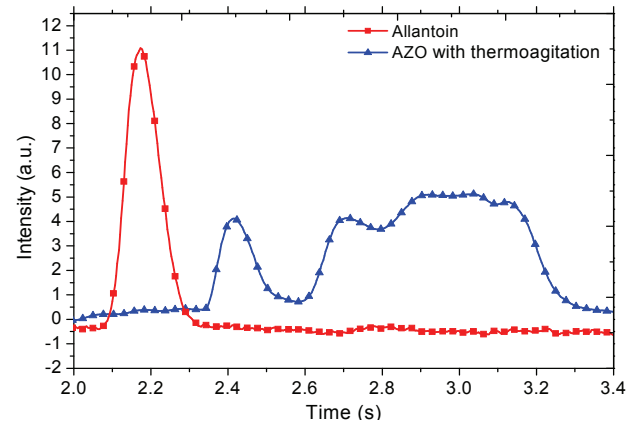

Figure 4: Chromatogram of the samples that suffered degradation with the reaction time of $20 \mathrm{~min}$.

It can be observed that the $\beta$-PVDF films with ITO electrodes did not influence the analyses results. The opposite happens when the electrodes of the $\beta$-PVDF film include aluminium, even in small concentrations, like the transparent conductive AZO.

\section{CONCLUSIONS}

The application of acoustic thermoagitation through the $\beta$-PVDF piezoelectric polymer is gainful when fluids need to be mixed in a microfluidic device. It reduces the mixing time resulting in a fast, complete and homogeneous reaction of the reactants, improving the global performance of the analysis that is being performed.

With the optimization tests, it was proved that better mixing results were obtained with the electrical signal for actuation at the resonance frequency of the film and with an amplitude of $10 \mathrm{~V}$. Moreover, it was demonstrated that the improvement of the mixture is mainly due to the heating of the solution.

In the degradation study, it was verified that, for all electrodes, the ITO is the one which does not degrade in contact with the uric acid kit.

\section{ACKNOWLEDGEMENTS}

Work supported by the Portuguese Science Foundation (grants PTDC/BIO/70017/2006, PTDC/CTM/69362/2006).

\section{REFERENCES}

Auroux, P., Iossifidis, D., Reyes, D. R., Manz, A., 2002, Micro Total Analysis Systems: Analytical Standard Operations and Applications. Anal. Chem., 74, p. 2637-2652.

Reyes, D. R., Iossifidis, D., Auroux, P., Manz, A., 2002, Micro Total Analysis Systems. 1. Introduction, Theory, and Technology. Anal. Chem., 74, p. 26232636.

Rife, J. C., et. al., 2000, Miniature Valveless Ultrasonic Pumps and Mixers. Sensors and Actuators B., 86, p. 135-140.

Ottino, J. M., Wiggins, S., 2004, Introduction: Mixing in Microfluidics. Phil. Trans. R. Soc. Lond. A. 362, p. 923-935.

Lanceros-Mendez, S., Sencadas, V., Gregorio Filho, R., Portuguese patent n. ${ }^{\circ} 103318$.

Thomas, M., 1999, Ultraviolet and visible spectroscopy. Analytical Chemistry by Open Learning, p. 2-47.

Frampton, K. D., Minor, K., Martin, S., 2004, Acoustic streaming in micro-scale cylindrical channels. Applied Acoustic, Vol. 65, p. 1121-1129.

Brown, L. F., 1992, Ferroelectric Polymers: current and future ultrasound applications. In IEEE Ultrason. Symp. Proc. p. 539-550.

Foster, F. S., 2000, A history of medical and biological imaging with polyvinylidene fluoride (PVDF transducers. In IEEE Transactions on Ultrasonics, Ferroelectrics and Frequency Control. Vol. $47 \mathrm{~N}^{\mathrm{o}} 6$.

Eiras, J. A., 2007, Piezoelectric Materials. Departamento de Física, Universidade Federal de São Carlos, Brasil. 